\title{
Führung und Team
}

\author{
Beate Fietze $\cdot$ Heidi Möller
}

Online publiziert: 15 . April 2020

(C) Springer Fachmedien Wiesbaden GmbH, ein Teil von Springer Nature 2020

Die Entstehung dieser Ausgabe der OSC steht unter dem Eindruck der CoronaPandemie. Wir erleben ein noch nie dagewesenes Sozialexperiment, das sich zuvor in seinen globalen Ausmaßen niemand hätte vorstellen können. Das ökonomische, soziale und kulturelle Leben wird auf unbestimmte Zeit auf das Allernotwendigste „heruntergefahren“: Die Bewegungsfreiheiten über die Staatsgrenzen hinweg werden stark eingeschränkt, die Flugzeuge bleiben am Boden, die Produktion wird gedrosselt, die sozialen Kontakte stehen unter Abstandsgebot.

Gegenwärtig wird der gesellschaftliche Diskurs über die Krise vorrangig von Virologen und Epidemiologen bestritten. Deren Problemdefinitionen und Situationsdeutungen wurden von den Akteuren des politischen Systems sehr schnell als Grundlage des eigenen Handelns übernommen. Die Mediziner selbst hingegen betonen die Begrenztheit ihrer fachlichen Zuständigkeit und fordern die Politiker auf, weitere Disziplinen heranzuziehen und ihre genuin politische Verantwortung zu übernehmen. Bisher werden die relevanten Fragen der ökonomischen, sozialen und kulturellen Folgekosten der Krisenbewältigung nicht ausreichend behandelt: Wie lange lassen sich ganze Volkswirtschaften durch noch so große staatliche Finanzzuschüsse in eine Art künstliches Koma versetzen? Welche politischen Folgen entstehen für die „Weltgesellschaft“"? Welche Teilgruppen der Bevölkerungen tragen langfristig den größten Schaden? Welche Einschränkungen der individuellen Freiheitsrechte sind angemessen oder auch nur temporär hinnehmbar? Welche ethischen

Dr. B. Fietze $(\bowtie)$

Institut an der Humboldt-Universität zu Berlin, artop GmbH, Christburger Str. 4, 10405 Berlin,

Deutschland

E-Mail: fietze@artop.de

Prof. Dr. H. Möller

Institut für Psychologie, Universität Kassel, Holländische Straße 36-38, 34127 Kassel, Deutschland

E-Mail: heidi.moeller@uni-kassel.de 
Dilemmata werden noch auftreten, zusätzlich zu der Triage am Krankenbett? Vor allem aber: Wie lassen sich überhaupt in diesen Dimensionen die langfristigen Risiken abschätzen und die „Fahrt auf Sicht“ einer iterativen politischen Prozesssteuerung mit einer langfristigen gesamtpolitischen Strategie verbinden? Die Erfahrungen in der Krise werden wohl zu veränderten gesellschaftlichen Selbstwahrnehmungen führen und möglicherweise - je nach Dauer der Krise - als formative Phase wirksam werden, die neue gesellschaftliche Wertorientierungen, Positionierungen, Bündnisse oder aber auch neue Konfliktlinien hervorbringt. Die Resultate lassen sich heute noch nicht absehen und müssen gerade deshalb in der gesellschaftlichen Reflexion und Diskussion darüber antizipiert werden.

Auch für die Berater/innen hat die Krise tiefgreifende Auswirkungen. Die ökonomischen Folgen des ,shut down“ und das rapide Wegbrechen der Finanzmittel in fast allen Branchen wirken sich direkt und ohne Zeitverzug auf die Nachfrage von Beratungsangeboten aus. Das trifft die Supervisor/innen, die Coaches und die Organisationsentwickler/innen in besonderer Weise, die mehrheitlich freiberuflich als Solo-Selbstständige arbeiten. Allerdings treffen nicht nur die ökonomischen Verwerfungen die Berater/innen hart. Die Beratungsform der ,,personenbezogenen Prozessberatung" lebt von der persönlichen Begegnung. Dies ist unter dem Gebot der Stunde des „social distancing“ nicht mehr in der gewohnten Weise möglich. Auch wenn in unserem Feld inzwischen virtuelle und ,blended“ Beratungsformate Einzug gehalten haben, werden doch bis heute die meisten Beratungen in Face-toFace-Settings durchgeführt (Bachmann und Fietze 2018). Die erzwungene physische Abstandnahme forciert die Digitalisierung in allen Bereichen. Innerhalb kürzester Zeit hat sich die gesellschaftliche Kommunikation in den virtuellen Raum der Videokonferenzen verlagert. Davon ist die Beratung nicht ausgenommen. Durch die verstärkte Nutzung bereits vorhandener virtueller Beratungsformate und die Entwicklung neuer Möglichkeiten wird sich die Praxis von Coaching, Supervision und Organisationsberatung verändern.

Unabhängig jedoch davon, inwieweit bisher die Beratungspraxis selbst in digitalen Formaten durchgeführt wird: Die digitale Transformation, die Innovationen der Arbeitswelt und die veränderten Anforderungen an die vorrangig arbeitsweltliche Beratung ist seit geraumer Zeit ein wichtiger Gegenstand der Diskussion. Eines der mit der digitalen Transformation verbundenen Themen ist die Beschäftigung mit den innovativen Kooperationsformen des „New Work“, die die Teamarbeit in den Fokus gerückt haben - dies war der Themenschwerpunkt im Heft 2/2019 in dieser Zeitschrift. Besonders mit den Konzepten des selbstorganisierten Teams der neueren Managementtheorien werden hohe Erwartungen verknüpft (Laloux 2015; Oestreich und Schröder 2017). Hier gibt es teilweise auch romantisierende Vorstellungen, die ihre Pointe darin haben, auf Führung vollständig verzichten zu können. Aber nicht alle Organisationen können dem Modell des ,Scrum Master“ folgen, und auch in selbstorgansierten Teams findet Führung statt (Wimmer 2019). Wir wollen deshalb in diesem Heft das Verhältnis von Führung und Team aus verschiedenen Perspektiven in den Blick nehmen.

Ausgehend von einer grundlegenden Klärung der Begriffe Gruppe, Thema und Führung untersucht Wolfgang Scholl das Verhalten der Mitglieder innerhalb eines Teams und zeigt in empirisch-analytischen Tiefenbohrungen, auf welche Weise neue 
Balancen von Gruppe und Führung jenseits der üblichen Führungskonzepte möglich werden. Dabei können die innovativen Führungskonzepte von heute aus einer Forschungstradition schöpfen, die bis in die Mitte des 20sten Jahrhunderts zurückreicht. Erst in der gegenwärtigen Wissensgesellschaft treffen jedoch die Führungskonzepte der transformationalen Führung wie Empowerment oder geteilte Führung des New Team-Work auf eine breite Resonanz.

Till Novotny identifiziert einen Paradigmenwechsel in der Führungskultur des Top-Managements. Während die ,,alte Leistungslogik“ auf dem Gedanken der Bestenauslese individueller Leistungsträger beruht, richtet sich die „,neue Leistungslogik“ auf die Qualität der Zusammenarbeit der Vorstandmitglieder. Der Beitrag analysiert die Eigenschafen, Arbeitsmodi und nötigen Teamkompetenzen erfolgreicher Teams an der Spitze, um in Zeiten unwägbarer Entscheidungsrisiken als Team resilient agieren zu können. Abschließend zeigt der Autor realistische Wege für die Begleitung von Teamentwicklungsprozessen auf Vorstandsebene.

Auch Sebastian Kunert und Sarah Dittmann stellen das Team ins Zentrum ihrer Betrachtung: Mit Hilfe einer Online-Befragung analysieren sie in einem zweistufigen quantitativen Verfahren den Einfluss des Teamklimas auf den Erfolg der Organisation. Während die erste Teilstudie wie erwartet die positive Bedeutung des Teamklimas bestätigt, kommt die zweite Teilstudie, die die Gruppenebene mit der Organisationskultur, dem Führungsrepertoire und der Mitarbeit/innen-Motivation vergleicht, zu einem anderen Ergebnis. Hier treten interessanterweise statt des Teamklimas die Effekte der Organisations- und Individualebene für den Organisationserfolg in den Vordergrund. Diese Ergebnisse führen zu neuen Perspektiven für die Beratung von Führungskräften.

Der Beitrag von Christina Lange und Thomas Webers knüpft an den Diskurs des vorletzten Heftes an, die umstrittene Debatte über die Führungskraft als Coach. Viele Führungskräfte lieben es, sich als Coach zu verstehen, aber was sagen deren Mitarbeiter/innen und die Mitarbeiter/innenvertretungen dazu? Die Autor/innen haben die Betroffenen qualitativ befragt und kommen aus deren Perspektive ebenfalls zu einer recht kritischen Sicht der Rollenüberfrachtung und -vermischung.

Nachdem jahrzehntelang in der Arbeits- und Organisationspsychologie Motivationsforschung mit Selbstauskünften betrieben wurde, hat sich aktuell eine Trendwende ergeben. Selbstauskünfte konnten zuvor nur die Konstruktionen eigener, kognitiv zugänglicher Aussagen über das, was jemanden antreibt, erfassen. Durch die Hinwendung zu impliziten Motivationsmustern wird in der Forschung auf die Befunde reagiert, dass sich Motivation schon recht früh, in vorsprachlichen Zeiten entwickeln. Nadia Hamdi Bek arbeitet die Bedeutung impliziter Motivationsmuster im Management-Coaching heraus und markiert deren Stellenwert.

In unseren offenen Beiträgen starten wir mit einer empirischen Untersuchung des Coachings mit Gründer/innen. Isabell Diermann und Isabel Meyer zu Riemsloh haben zahlreiche Interviews mit Gründungs-Coaches und deren Klient/innen geführt und arbeiten den Unterschied zu üblichen Coachingformaten heraus. Die Rolle, die Anlässe und die Themen des Gründungsprozesses ordnen sie in einem Bereich des Zwischenraums Experten- versus Prozessberatung zu. Für das Coaching als Unterstützungsformat für Gründer/innen wird von den Autorinnen eine gute Grundlegung bereitgestellt. 
Alexander Berresheim und Ruthild Vaihinger führen uns in eine besondere Arbeitsaufgabe ein: die Beratung bei der Polizei. Den Autoren gelingt es, die Leser/ innen in diese spezielle Arbeitswelt mitzunehmen und wichtige Implikationen für das Training und die Beratung herauszuarbeiten. Für die Arbeit in diesem Feld sind Kenntnisse des Berufshabitus und der Organisationskultur unabdingbar, um für Angebote der Supervision, des Coachings und der Organisationsberatung anschlussfähig zu sein.

In unserer Rubrik Diskurs geschäftigen wir uns mit dem heiß diskutierten Thema der narzisstischen Führungskräfte. Über Thomas Middelhoff wurde schon viel (auch literarisch) geschrieben, als er noch als der deutsche Starmanager war. Herbert Csef nimmt sich nun der Verarbeitung des Niedergangs von Thomas Middelhoff an, nachdem er 2014 zu drei Jahren Haft verurteilt wurde. Seine beiden selbst verfassten Biografien muten nun nicht minder narzisstisch an: nur mit umgekehrten Vorzeichen. Die Nummer 1 im Leiden, den Schuldgefühlen und der Selbstzerknirschung zu sein, ist nichts weiter als die Schattenseite eines malignen Narzissmus.

Sandra Schiemann und Eva Jonas von der Universität Salzburg geben einen Überblick zur internationalen Forschung zur ,dunklen Triade“ (Narzissmus, Machiavellismus und Psychopathie). Sie geben Antwort auf die Frage, mit welchem Führungsverhalten diese Persönlichkeitsprofile einhergehen. Es wird aufgezeigt, welchen Schaden die Mitarbeiter/innen nehmen können und welche Risiken für die Organisation entstehen. Präventive Vorschläge sind dem Beitrag ebenso zu entnehmen wie eine Einbettung der Phänomene in die jeweilige Organisationskultur.

\section{Literatur}

Bachmann, T., \& Fietze, B. (2018). Die Digitalisierung von Coaching. Gedanken aus der Perspektive teilnehmender Beobachtung. Organisationsberatung, Supervision, Coaching, 25(3), 3-18.

Laloux, F. (2015). Reinventing Organization. Ein Leitfaden zur Gestaltung sinnstiftender Formen der Zusammenarbeit. München: Vahlen.

Oestereich, B., \& Schröder, C. (2017). Das kollegial geführte Unternehmen. Ideen und Praktiken für die agile Organisation von morgen. München: Vahlen.

Wimmer, R. (2019). Agilität, Ambidextrie und organisationale Veränderungskompetenz. Rudi Wimmer über Erbe und Zukunft des Change-Managements. Gruppe. Interaktion. Organisation. Zeitschrift für Angewandte Organisationspsychologie, 50(2), 211-216. 